\title{
ON SOLVABILITY OF A COUPLED HYBRID SYSTEM OF QUADRATIC FRACTIONAL INTEGRAL EQUATIONS
}

\author{
KAZEM GHANBARI AND YOUSEF GHOLAMI
}

\begin{abstract}
Of concern is studying solvability of the hybrid systems of quadratic fractional integral equations. To this aim applying hybrid fixed point theory due to Dhage, existence of at least one positive solution for mentioned systems via so called D-Lipschitzian mappings will be concluded. We illustrate the obtained results by presenting an example.
\end{abstract}

\section{Introduction}

The fractional calculus is the theory of arbitrary order integration and differentiation that generalizes the integer order ones in classic differential calculus. Not only in theoretical manner but also as a result of more accurate description of real world phenomena in comparison with classic differential calculus, we can observe the boom of development of theory of fractional calculus in less than three decades in almost whole sciences related to mathematics such as biosciences, medicine, engineering, economy and so on. More details and applications can be found in the monographs and papers [26], [23], [1]-[12], [14]-[20]. On the other hand the theory of integral equations by itself has been introduced as full applicable theory in mathematics and great theories such as geomagnetic theory, transport theory, mechanics and so forth. So we can conclude that combination of fractional calculus and integral equations may introduce more effective tool for analysis and description of topics mentioned above. In this way more interesting applications can be found in references [25], [27] and references cited therein. Let us now introduce in the sequel the main motivation for preparing this paper.

The authors in [22], by means of Dhage hybrid fixed point theory obtained periodic solutions of integral equation

$$
x(t)=\sum_{i=1}^{n} f_{i}\left(t, x\left(a_{i}(t)\right)\right) \cdot \int_{\mathbb{R}} k_{i}(t, s) g_{i}\left(s, x\left(b_{i}(s)\right)\right) d s .
$$

Received June 4, 2015, accepted January 6, 2016.

2010 Mathematics Subject Classification. Primary: 26A33, 45G15, Secondary: 34B18, 47H10.

Key words and phrases. Fractional calculus, integral equations, fixed point theory, positive solution.

Corresponding author: Kazem Ghanbari. 
The authors in [13], considered the fractional order integral equation

$$
x(t)=f(t, x(t))+g(t, x(t)) \int_{0}^{t} \frac{(t-s)^{\alpha-1}}{\Gamma(\alpha)} u(s, x(s)) d s, \quad t \in J=[0,1], \alpha>0,
$$

and via above mentioned theory concluded the existence of at least one solution.

In this paper we consider the coupled hybrid system of quadratic fractional integral equations

$$
\left\{\begin{array}{l}
u(t)=\sum_{i=1}^{n}\left\{k_{1, i}(t, u(t), v(t))+g_{1, i}(t, u(t), v(t)) \cdot \int_{0}^{t} \frac{(t-s)^{\alpha_{i}-1}}{\Gamma\left(\alpha_{i}\right)} f_{1, i}(s, u(s), v(s)) d s\right\}, \\
v(t)=\sum_{j=1}^{n}\left\{k_{2, j}(t, u(t), v(t))+g_{2, j}(t, u(t), v(t)) \cdot \int_{0}^{t} \frac{(t-s)^{\beta_{j}-1}}{\Gamma\left(\beta_{j}\right)} f_{2, j}(s, u(s), v(s)) d s\right\},
\end{array}\right.
$$

where $t \in \mathbb{J}=[0, T], T, \alpha_{i}, \beta_{j} \in \mathbb{R}^{+}, i, j=1,2, \ldots, n$.

Assume that the following hypotheses are satisfied throughout this paper.

$\left(A_{1}\right) k_{1, i} \in C\left(\sqrt{ } \times \mathbb{R}^{2}, \mathbb{R}^{+}\right)$for $i=1,2, \ldots, n$ and there exist positive constants $L_{1, k_{1, i}}, L_{2, k_{1, i}}, \rho_{k_{1, i}}$ with $L_{1, k_{1, i}} \leq L_{2, k_{1, i}}$ such that

$$
\left\|k_{1, i}\left(t, u_{1}, .\right)-k_{1, i}\left(t, u_{2}, .\right)\right\|_{E} \leq \frac{1}{2 n} \frac{L_{1, k_{1, i}} \ln \left(1+\left\|u_{1}-u_{2}\right\|_{E}\right)}{L_{2, k_{1, i}}+\ln \left(1+\left\|u_{1}-u_{2}\right\|_{E}\right)}, t \in \mathbb{J}, u_{1}, u_{2} \in C(\mathbb{R}),
$$

where $E=C(\mathbb{J}, \mathbb{R})$ is a Banach space endowed with the max-norm, also assume that

$$
\sup k_{1, i}(t, u, v)=\rho_{k_{1, i}}, \quad i=1,2, \ldots, n, t \in \mathbb{J}, u, v \in C(\mathbb{R})
$$

( $\left.A_{2}\right) g_{1, i} \in C\left(\mathbb{J} \times \mathbb{R}^{2}, \mathbb{R}^{+}\right)$for $i=1,2, \ldots, n$ and there exist positive constants $L_{1, g_{1, i}}, L_{2, g_{1, i}}, \rho_{g_{1, i}}$ with $L_{1, g_{1, i}} \leq L_{2, g_{1, i}}$ such that

$$
\left\|g_{1, i}\left(t, u_{1}, .\right)-g_{1, i}\left(t, u_{2}, .\right)\right\|_{E} \leq \frac{1}{2 n M_{i}} \frac{L_{1, g_{1, i}} \ln \left(1+\left\|u_{1}-u_{2}\right\|_{E}\right)}{L_{2, g_{1, i}}+\ln \left(1+\left\|u_{1}-u_{2}\right\|_{E}\right)}, t \in \mathbb{J}, u_{1}, u_{2} \in C(\mathbb{R}),
$$

where $M_{i}=\left\|I_{0^{+}}^{\alpha_{i}} f_{1, i}(., u, v)\right\|$, also suppose that

$$
\sup _{1, i}(t, u, v)=\rho_{g_{1, i}}, \quad i=1,2, \ldots, n, t \in \mathbb{J}, u, v \in C(\mathbb{R})
$$

( $\left.A_{3}\right) f_{1, i}, f_{2, j} \in C\left(\mathbb{J} \times \mathbb{R}^{2}, \mathbb{R}^{+}\right)$for $i, j=1,2, \ldots, n$ and

$$
\sup f_{1, i}(t, u, v)=\theta_{1, i}, \quad \sup f_{2, j}(t, u, v)=\theta_{2, j}, \quad i, j=1,2, \ldots, n, t \in \mathbb{J}, u, v \in C(\mathbb{R}) .
$$

( $\left.A_{4}\right)$ Replacing $i$ with $j$ and $u$ with $v$ also $\alpha$ with $\beta$ in conditions $\left(A_{1}\right),\left(A_{2}\right)$ the corresponding conditions will be satisfied for $k_{2, j}, g_{2, j}$ for $j=1,2, \ldots, n$ in hybrid system (1.1). 


\section{Preliminaries}

This section contains two steps. First we represent some concepts from fractional calculus that will be needed in the sequel and in second one in preparatory manner we will briefly overview the hybrid fixed point theory due to B. C. Dhage.

Definition 2.1 ([23]). The fractional Riemann-Liouville integral of order $\alpha>0$ for function $f \in L^{1}(0, \infty)$ is defined as

$$
I_{0^{+}}^{\alpha} f(t)=\frac{1}{\Gamma(\alpha)} \int_{0}^{t}(t-s)^{\alpha-1} f(s) d s,
$$

where $\Gamma(\alpha)=\int_{0}^{\infty} s^{\alpha-1} e^{-s} d s$ denotes the Euler gamma function.

Definition 2.2 ([23]). The fractional Riemann-Liouville derivative of order $\alpha>0$ for function $f \in L^{1}(0, \infty)$ is given by

$$
D_{0^{+}}^{\alpha} f(t)=\frac{1}{\Gamma(n-\alpha)}\left(\frac{d^{n}}{d t^{n}}\right) \int_{0}^{t}(t-s)^{n-\alpha-1} f(s) d s,
$$

where $n=[\alpha]+1$.

Lemma 2.3 ([26]). Let $\alpha>0$ then

(i) If $\mu>-1, \mu \neq \alpha-i$ with $i=1,2, \ldots,[\alpha]+1$ and $t>0$, then $D_{0^{+}}^{\alpha} t^{\mu}=\frac{\Gamma(\mu+1)}{\Gamma(\mu-\alpha+1)} t^{\mu-\alpha}$.

(ii) $D_{0^{+}}^{\alpha} t^{\alpha-i}=0$, for $i=1,2, \ldots,[\alpha]+1$.

(iii) If $u \in L^{1}(0, \infty)$, then $D_{0^{+}}^{\alpha} I_{0^{+}}^{\alpha} u(t)=u(t), \quad I_{0^{+}}^{\alpha} D_{0^{+}}^{\alpha} u(t)=u(t)+\sum_{i=1}^{n} c_{i} t^{\alpha-i}$.

(iv) $D_{0^{+}}^{\alpha} u(t)=0 \Leftrightarrow u(t)=\sum_{i=1}^{n} c_{i} t^{\alpha-i}$,

where $c_{i} \in \mathbb{R}, n=[\alpha]+1$. We notice that replacing $\alpha$ with $-\alpha$ in (i), gives us fractional integration of power functions.

In what follows we will apply the Banach space $\left(\mathfrak{B},\|\cdot\|_{\mathfrak{B}}\right)$ :

$$
\begin{aligned}
\mathfrak{B} & =E \times E, \quad E=\{u \mid u \in C(\mathbb{J}, \mathbb{R})\}, \\
\|(u, v)\|_{\mathfrak{B}} & =\|u\|_{E}+\|v\|_{E}, \quad\|u\|_{E}=\max _{t \in \mathbb{J}}\{u(t) \mid u \in E\} .
\end{aligned}
$$

Define set $S \subset \mathfrak{B}$ as

$$
\begin{aligned}
S & =\left\{(u, v) \in \mathfrak{B} \mid u(t), v(t) \geq 0, t \in \mathbb{J},\|(u, v)\|_{\mathfrak{B}} \leq r\right\}, \\
& =\left\{u, v \in E \mid u(t), v(t) \geq 0, t \in \mathbb{J},\|u\|_{E}+\|v\|_{E} \leq r\right\} .
\end{aligned}
$$

Definition 2.4. We define the integral operators $T_{1}, T_{2}: E \rightarrow E$ by

$$
\begin{aligned}
& T_{1, u}(t)=\sum_{i=1}^{n}\left\{k_{1, i}(t, u(t), v(t))+g_{1, i}(t, u(t), v(t)) \cdot \int_{0}^{t} \frac{(t-s)^{\alpha_{i}-1}}{\Gamma\left(\alpha_{i}\right)} f_{1, i}(s, u(s), v(s)) d s\right\}, \\
& T_{2, v}(t)=\sum_{j=1}^{n}\left\{k_{2, j}(t, u(t), v(t))+g_{2, j}(t, u(t), v(t)) \cdot \int_{0}^{t} \frac{(t-s)^{\beta_{j}-1}}{\Gamma\left(\beta_{j}\right)} f_{2, j}(s, u(s), v(s)) d s\right\} .
\end{aligned}
$$


Now we can define the operator $\mathfrak{T}: \mathfrak{B} \rightarrow \mathfrak{B}$ as follows

$$
\mathfrak{T}(u, v)=\left(T_{1, u}, T_{2, v}\right)
$$

Definition 2.5 ([1]). Let $X$ be a normed vector space. A mapping $T: X \rightarrow X$ is said to be DLipschitzian, provided there exists a continuous and nondecreasing function $\psi_{T}: \mathbb{R}^{+} \rightarrow \mathbb{R}^{+}$ such that for $x, y \in X$

$$
\|T x-T y\| \leq \psi_{T}(\|x-y\|), \quad \psi_{T}(0)=0 .
$$

The function $\psi_{T}$ is called a D-function of $T$ on $X$.

Remark 2.6 ([24]). If $\phi, \psi: \mathbb{R}^{+} \rightarrow \mathbb{R}^{+}$are two D-functions, then i) $\phi+\psi$, ii) $\lambda \phi$, for $\lambda>0$, and iii) $\phi о \psi$ are also D-functions on $\mathbb{R}^{+}$. Frequently used D-functions are $\phi(r)=k r$, with $k>0$, $\phi(r)=\frac{L r}{K+r}$, with $0 \leq L \leq K, \phi(r)=r-\ln (1+r), \phi(r)=\ln (1+r)$, etc. So we conclude that the inequalities in hypotheses $\left(A_{1}\right)-\left(A_{4}\right)$ are reasonable.

Definition 2.7 ([21]). Let $X$ be a normed space and suppose $S \subset X$. A finite set of $N$ balls $B\left(x_{n}, \epsilon\right)$ with $x_{n} \in X$ and $\epsilon>0$ is said to be a finite $\epsilon$-covering of $S$, provided that every element of $S$ lies inside one of the balls $B\left(x_{n}, \epsilon\right)$, i.e.

$$
S \subset \bigcup_{n=1}^{N} B\left(x_{n}, \epsilon\right) .
$$

The set of centers $\left\{x_{n}\right\}$ of a finite $\epsilon$-covering is called a finite $\epsilon$-net for $S$.

Definition 2.8 ([21]). Let $X$ be a normed space. A set $S \subset X$ is said to be a Totally Bounded if and only if it has a finite $\epsilon$-covering for every $\epsilon>0$.

Theorem 2.9 (Hausdorff compactness criterion [21]). Assume that X be a normed space. A set $S \subset X$ is compact if and only if it is closed and totally bounded.

Theorem 2.10 (Dhage fixed point theorem [1]). Assume that $S$ be a nonempty closed convex and bounded subset of Banach algebra $X$. Let $A, C: X \rightarrow X$ and $B: S \rightarrow X$ be three operators with the following properties:

(i) A,C are D-Lipschitzian with D-functions $\phi_{A}$ and $\phi_{C}$ respectively.

(ii) B is completely continuous.

(iii) $x=A x+C x B y \Rightarrow x \in S$, for all $y \in S$.

(iv) $\phi_{A}(r)+M \phi_{C}(r)<r$, for $r>0$ where $M=\|B(S)\|$.

Then the equation $A x+C x B x=x$ has a solution in $S$. 


\section{Main results}

Theorem 3.1. Suppose that the conditions $\left(A_{1}\right)-\left(A_{4}\right)$ hold. Then the fractional coupled system (1.1) has at least one positive solution in $S$.

Proof. We are going to the represent of the proof in three steps as follows:

$\left(S_{1}\right)$ Suppose that

$$
\begin{aligned}
& A_{1, i} u(t)=\sum_{i=1}^{n} k_{1, i}(t, u(t), v(t)), \\
& C_{1, i} u(t)=g_{1, i}(t, u(t), v(t)), i=1,2, \ldots, n, \\
& B_{1, i} u(t)=\int_{0}^{t} \frac{(t-s)^{\alpha_{i}-1}}{\Gamma\left(\alpha_{i}\right)} f_{1, i}(s, u(s), v(s)) d s, i=1,2, \ldots, n, \\
& A_{2, j} v(t)=\sum_{j=1}^{n} k_{2, j}(t, u(t), v(t)), \\
& C_{2, j} v(t)=g_{2, j}(t, u(t), v(t)), j=1,2, \ldots, n, \\
& B_{2, j} v(t)=\int_{0}^{t} \frac{(t-s)^{\beta_{j}-1}}{\Gamma\left(\beta_{j}\right)} f_{2, j}(s, u(s), v(s)) d s, j=1,2, \ldots, n .
\end{aligned}
$$

Define

$$
\begin{aligned}
A_{1, i, 2, j}(u, v)(t) & =\left(\begin{array}{l}
\sum_{i=1}^{n} k_{1, i}(t, u(t), v(t)) \\
\sum_{j=1}^{n} k_{2, j}(t, u(t), v(t))
\end{array}\right), \\
C_{1, i, 2, j}(u, v)(t) & =\left(\begin{array}{cc}
g_{1, i}(t, u(t), v(t)) & 0 \\
0 & g_{2, j}(t, u(t), v(t))
\end{array}\right), \quad i, j=1,2, \ldots, n, \\
B_{1, i, 2, j}(u, v)(t) & =\left(\begin{array}{cc}
\int_{0}^{t} \frac{(t-s)^{\alpha_{i}-1}}{\Gamma\left(\alpha_{i}\right)} f_{1, i}(s, u(s), v(s)) d s \\
\int_{0}^{t} \frac{(t-s)^{\beta_{j}-1}}{\Gamma\left(\beta_{j}\right)} f_{2, j}(s, u(s), v(s)) d s
\end{array}\right), \quad i, j=1,2, \ldots, n .
\end{aligned}
$$

Now we can transform operator $\mathfrak{T}(u, v)$ defined by (2.5) to the operator

$$
\mathfrak{T}(u, v)(t)=A_{1, i, 2, j}(u, v)(t)+\sum_{i} \sum_{j} C_{1, i, 2, j}(u, v)(t) B_{1, i, 2, j}(u, v)(t),
$$

such that above double summation acts on $(i, j)=(1,1),(2,2), \ldots,(n, n)$.

Indeed we shall show in this step that $\mathfrak{T}(u, v)$ is a nonlinear D-Lipschitzian with Dfunction

$$
\psi_{u, v}=\sum_{i=1}^{n} \psi_{k_{1, i}}+\sum_{j=1}^{n} \psi_{k_{2, j}}+\sum_{i} \sum_{j}\left\{\psi_{g_{1, i}} M_{i}+\psi_{g_{2, j}} M_{j}\right\},
$$

where $(i, j)=(1,1),(2,2), \ldots,(n, n)$ and $\psi_{k_{1, i}}, \psi_{k_{2, j}}, \psi_{g_{1, i}}, \psi_{g_{2, j}}$ are D-functions corresponding to the nonlinear D-Lipschitzian $k_{1, i}, k_{2, j}, g_{1, i}, g_{2, j}$. To this aim by means of 
conditions $\left(A_{1}\right),\left(A_{4}\right)$ we have

$$
\begin{aligned}
\left\|A_{1, i, 2, j}\left(u_{1}, v_{1}\right)-A_{1, i, 2, j}\left(u_{2}, v_{2}\right)\right\|_{\mathfrak{B}} \\
\quad \leq \sum_{i=1}^{n}\left\|k_{1, i}\left(., u_{1}, v_{1}\right)-k_{1, i}\left(., u_{2}, v_{2}\right)\right\|_{E}+\sum_{j=1}^{n}\left\|k_{2, j}\left(., u_{1}, v_{1}\right)-k_{2, j}\left(., u_{2}, v_{2}\right)\right\|_{E} \\
\quad \leq \sum_{i=1}^{n} \frac{L_{1, k_{1, i}} \ln \left(1+\left\|u_{1}-u_{2}\right\|_{E}\right)}{2 n\left(L_{2, k_{1, i}}+\ln \left(1+\left\|u_{1}-u_{2}\right\|_{E}\right)\right)}+\sum_{j=1}^{n} \frac{L_{1, k_{2, j}} \ln \left(1+\left\|v_{1}-v_{2}\right\|_{E}\right)}{2 n\left(L_{2, k_{2, j}}+\ln \left(1+\left\|v_{1}-v_{2}\right\|_{E}\right)\right)} \\
\quad \leq \frac{\ln \left(1+\left\|u_{1}-u_{2}\right\|_{E}\right)}{2}+\frac{\ln \left(1+\left\|v_{1}-v_{2}\right\|_{E}\right)}{2} \\
\quad \leq \frac{\ln \left(1+\left\|\left(u_{1}, u_{2}\right)-\left(v_{1}, v_{2}\right)\right\|_{\mathfrak{B}}\right)}{2}+\frac{\ln \left(1+\left\|\left(u_{1}, u_{2}\right)-\left(v_{1}, v_{2}\right)\right\|_{\mathfrak{B}}\right)}{2} \\
\quad=\ln \left(1+\left\|\left(u_{1}, u_{2}\right)-\left(v_{1}, v_{2}\right)\right\|_{\mathfrak{B}}\right) .
\end{aligned}
$$

Thus $k_{1, i}, k_{2, j}$ are two D-Lipschitzian with corresponding D-functions

$$
\psi_{k_{1, i}}(r)=\frac{L_{1, k_{1, i}} \ln (1+r)}{2 n\left(L_{2, k_{1, i}}+\ln (1+r)\right)}, \quad \psi_{k_{2, j}}(r)=\frac{L_{1, k_{2, j}} \ln (1+r)}{2 n\left(L_{2, k_{2, j}}+\ln (1+r)\right)}, i, j=1,2, \ldots, n .
$$

Equivalently we have been proved that $A_{1, i, 2, j}(u, v)$ is a D-Lipschitzian with corresponding D-function

$$
\psi_{A_{1, i, 2, j}}(r)=\left(\sum_{i=1}^{n} \psi_{k_{1, i}}+\sum_{j=1}^{n} \psi_{k_{2, j}}\right) r .
$$

Similarly by means of conditions $\left(A_{2}\right),\left(A_{4}\right)$ we conclude that

$$
\begin{aligned}
\sum_{i} \sum_{j} & \left\|C_{1, i, 2, j} B_{1, i, 2, j}\left(u_{1}, v_{1}\right)-C_{1, i, 2, j} B_{1, i, 2, j}\left(u_{2}, v_{2}\right)\right\|_{\mathfrak{B}} \\
\leq & \sum_{i} \sum_{j}\left\{\left\|g_{1, i}\left(., u_{1}, v_{1}\right)-g_{1, i}\left(., u_{2}, v_{2}\right)\right\|_{E}\left\|I_{0^{+}}^{\alpha_{i}} f_{1, i}\right\|_{E}+\| g_{2, j}\left(., u_{1}, v_{1}\right)\right. \\
& \left.-g_{2, j}\left(., u_{2}, v_{2}\right)\left\|_{E}\right\| I_{0^{+}}^{\beta_{j}} f_{2, j} \|_{E}\right\} \\
\leq & \sum_{i} \sum_{j}\left\{\frac{L_{1, g_{1, i}} \ln \left(1+\left\|u_{1}-u_{2}\right\|_{E}\right) M_{i}}{2 n M_{i}\left(L_{2, g_{1, i}}+\ln \left(1+\left\|u_{1}-u_{2}\right\|_{E}\right)\right)}+\frac{L_{1, g_{2, j}} \ln \left(1+\left\|v_{1}-v_{2}\right\|_{E}\right) M_{j}}{2 n M_{j}\left(L_{2, g_{2, j}}+\ln \left(1+\left\|v_{1}-v_{2}\right\|_{E}\right)\right)}\right\} \\
\leq & \frac{\ln \left(1+\left\|u_{1}-u_{2}\right\|_{E}\right)}{2}+\frac{\ln \left(1+\left\|v_{1}-v_{2}\right\|_{E}\right)}{2} \\
\leq & \frac{\ln \left(1+\left\|\left(u_{1}, u_{2}\right)-\left(v_{1}, v_{2}\right)\right\|_{\mathfrak{B}}\right)}{2}+\frac{\ln \left(1+\left\|\left(u_{1}, u_{2}\right)-\left(v_{1}, v_{2}\right)\right\|_{\mathfrak{B}}\right)}{2} \\
= & \ln \left(1+\left\|\left(u_{1}, u_{2}\right)-\left(v_{1}, v_{2}\right)\right\|_{\mathfrak{B}}\right) .
\end{aligned}
$$

for $(i, j)=(1,1),(2,2), \ldots,(n, n)$. Hence we deduce that both of operators $g_{1, i}, g_{2, j}$ are D-Lipschitzian with corresponding D-functions

$$
\psi_{g_{1, i}}(r)=\frac{L_{1, g_{1, i}} \ln (1+r)}{2 n M_{i}\left(L_{2, g_{1, i}}+\ln (1+r)\right)}, \quad \psi_{g_{2, j}}(r)=\frac{L_{1, g_{2, j}} \ln (1+r)}{2 n M_{j}\left(L_{2, g_{2, j}}+\ln (1+r)\right)}, i, j=1,2, \ldots, n .
$$


It can be that $\sum_{i} \sum_{j} C_{1, i, 2, j} B_{1, i, 2, j}(u, v)$ is a D-Lipschitzian with corresponding D-function

$$
\psi_{C_{1, i, 2, j}}(r)=\sum_{i} \sum_{j}\left[\psi_{g_{1, i}} M_{i}+\psi_{g_{2, j}} M_{j}\right] r,(i, j)=(1,1),(2,2), \ldots,(n, n) .
$$

At last, by means of (3.8)-(3.13) one can observe that $\mathfrak{T}(u, v)$ defined by (3.6) is a $\mathrm{D}$ Lipschitzian with corresponding D-function $\psi_{u, v}$ defined by (3.7). So $\left(S_{1}\right)$ is complete now.

$\left(S_{2}\right)$ In this step we must prove that the operator $B_{1, i, 2, j}(u, v)$ defined by (3.5) is completely continuous on $S$ defined by (2.3). In this way firstly applying the Hausdorff compactness criterion given by Theorem 2.9, we will prove that $S$ is a compact subset of Banach space $\mathfrak{B}$.

It is clear that $S \subset \mathfrak{B}$ is a cone in $\mathfrak{B}$. Let us define

$$
\begin{aligned}
& S_{u}=\left\{u \in E \mid\|u\|_{E} \leq \frac{r}{2}, t \in \mathbb{J}\right\}, \\
& S_{\nu}=\left\{v \in E \mid\|v\|_{E} \leq \frac{r}{2}, t \in \mathbb{J}\right\} .
\end{aligned}
$$

Clearly $S_{u}, S_{v}$ is closed. Therefore $S_{u}, S_{v}$ are complete. Thus as a result of equicontinuity of $u(t), v(t)$, the Arzela-Ascoli theorem implies that $S_{u}, S_{v}$ are relatively compact. Hence Theorem 2.9 ensures that $S_{u}, S_{v}$ are totally bounded. Thus via Definition 2.8 we conclude that there exist two finite $\epsilon$-coverings as

$$
\mathfrak{U}_{\epsilon}\left(u_{i}\right), \mathfrak{U}_{\epsilon}\left(v_{j}\right) \quad i=1,2,3, \ldots, l_{1}, j=1,2,3, \ldots, l_{2},
$$

such that

$$
\begin{aligned}
& S_{u} \subset \bigcup_{i=1}^{l_{1}} \mathfrak{U}_{\epsilon}\left(u_{i}\right), \\
& S_{v} \subset \bigcup_{j=1}^{l_{2}} \mathfrak{U}_{\epsilon}\left(v_{j}\right),
\end{aligned}
$$

where

$$
\begin{aligned}
& \mathfrak{U}_{\epsilon}\left(u_{i}\right)=\left\{u \in S_{u} \mid\left\|u-u_{i}\right\|_{E}<\epsilon\right\}, \\
& \mathfrak{U}_{\epsilon}\left(v_{j}\right)=\left\{v \in S_{\nu} \mid\left\|v-v_{j}\right\|_{E}<\epsilon\right\} .
\end{aligned}
$$

Define

$$
S_{i j}=\left\{(u, v) \in S_{u} \times S_{\nu} \mid u \in \mathfrak{U}_{\epsilon}\left(u_{i}\right), v \in \mathfrak{U}_{\epsilon}\left(v_{j}\right)\right\} .
$$

It is easy to see that $S \subset S_{u} \times S_{\nu} \subset \bigcup_{i, j} S_{i j}, 1 \leq i \leq l_{1}, 1 \leq j \leq l_{2}$.

In fact if we take $\left(u_{i j}, v_{i j}\right) \in S_{i j}$, then $S_{u} \times S_{v}$ can be covered by finite $4 \epsilon$-covering

$$
\mathfrak{U}_{4 \epsilon}\left(u_{i j}, v_{i j}\right)=\left\{(u, v) \in S_{u} \times S_{\nu} \mid\left\|(u, v)-\left(u_{i j}, v_{i j}\right)\right\|_{\mathfrak{B}}<4 \epsilon\right\} .
$$

In other means for every $(u, v) \in S_{u} \times S_{v}$, there exist indices $i, j$ such that

$$
u \in \mathfrak{U}_{\epsilon}\left(u_{i}\right), \quad v \in \mathfrak{U}_{\epsilon}\left(v_{j}\right) .
$$


Therefore

$$
\begin{aligned}
& \left|u-u_{i j}\right| \leq\left|u-u_{i}\right|+\left|u_{i}-u_{i j}\right|<\epsilon+\epsilon=2 \epsilon, \\
& \left|v-v_{i j}\right| \leq\left|v-v_{i}\right|+\left|v_{i}-v_{i j}\right|<\epsilon+\epsilon=2 \epsilon .
\end{aligned}
$$

(3.17) implies that $\left\|(u, v)-\left(u_{i j}, v_{i j}\right)\right\|_{\mathfrak{B}}<4 \epsilon$. Hence $S$ has a finite $4 \epsilon$-covering. Therefore using Theorem 2.9 we conclude that $S$ is compact.

Turning back to the definition of $B_{1, i, 2, j}(u, v)$ given by (3.5) and considering the condition $\left(A_{3}\right)$, we conclude that $B_{1, i, 2, j}(u, v)$ is continuous on $S$. Thus $B_{1, i, 2, j}(S)$ is completely continuous on $S$. This completes the $\left(S_{2}\right)$

$\left(S_{3}\right)$ In the last step we are going to show that if

$$
\begin{aligned}
& u_{*}=\sum_{i=1}^{n}\left\{k_{1, i}\left(., u_{*}, v\right)+g_{1, i}\left(., u_{*}, v\right) . I_{0^{+}}^{\alpha_{i}} f_{1, i}\left(., u_{*}, v\right)\right\}, \\
& v_{*}=\sum_{j=1}^{n}\left\{k_{2, j}\left(., u, v_{*}\right)+g_{2, j}\left(., u, v_{*}\right) . I_{0^{+}}^{\beta_{j}} f_{2, j}\left(., u, v_{*}\right)\right\},
\end{aligned}
$$

then $\left(u_{*}, v_{*}\right) \in S$ for all $(u, v) \in S$. By means of conditions $\left(A_{1}\right)-\left(A_{4}\right)$ and Applying Lemma 2.3 , it is easy to check that

$$
\begin{aligned}
& T_{1, u}(t) \leq \sum_{i=1}^{n} \frac{\Gamma\left(\alpha_{i}+1\right) \rho_{k_{1, i}}+\rho_{g_{1, i}} \theta_{1, i} T^{\alpha_{i}}}{\Gamma\left(\alpha_{i}+1\right)}=r_{1}, \\
& T_{2, v}(t) \leq \sum_{j=1}^{n} \frac{\Gamma\left(\beta_{j}+1\right) \rho_{k_{2, j}}+\rho_{g_{2, j}} \theta_{2, j} T^{\beta_{j}}}{\Gamma\left(\beta_{j}+1\right)}=r_{2} .
\end{aligned}
$$

On the other hand $\|\mathfrak{T}(u, v)\|_{\mathfrak{B}}=\left\|T_{1, u}\right\|_{E}+\left\|T_{2, v}\right\|_{E}$. So we have

$$
\|\mathfrak{T}(u, v)\|_{\mathfrak{B}} \leq r=2 \max \left\{r_{1}, r_{2}\right\} .
$$

Equivalently, we have proved that $\mathfrak{T}(S) \subset S$, that is if (3.18) be satisfied, then $\left(u_{*}, v_{*}\right) \in S$ for all $(u, v) \in S$. So $\left(S_{3}\right)$ is completed.

Since all of the conditions (i) - (iv) in Theorem 2.10 hold, then the coupled hybrid system of fractional quadratic integral equations (1.1) has at least one positive solution in $S$.

\section{An example}

Let us consider the coupled hybrid system of FQIEs

$$
\left\{\begin{array}{l}
u(t)=\sum_{i=1}^{n}\left\{k_{1, i}(t, u(t), v(t))+g_{1, i}(t, u(t), v(t)) \cdot \int_{0}^{t} \frac{(t-s)^{\alpha_{i}-1}}{\Gamma\left(\alpha_{i}\right)} f_{1, i}(s, u(s), v(s)) d s\right\}, \\
v(t)=\sum_{j=1}^{n}\left\{k_{2, j}(t, u(t), v(t))+g_{2, j}(t, u(t), v(t)) \cdot \int_{0}^{t} \frac{(t-s)^{\beta_{j}-1}}{\Gamma\left(\beta_{j}\right)} f_{2, j}(s, u(s), v(s)) d s\right\} .
\end{array}\right.
$$


Take $t \in \mathbb{J}=[0,4]$ and $\alpha_{i}=\frac{2 i-1}{2}, \beta_{j}=\frac{2 j+1}{4}$ for $i, j=1,2, \ldots, n$. Setting

$$
L_{p, k_{1, i}}=L_{p, k_{2, i}}=L_{p, g_{1, j}}=L_{p, g_{2, j}}=1, \quad p=1,2, i, j=1,2, \ldots, n,
$$

and

$$
\left\{\begin{array}{l}
k_{1, i}(t, u, v)=\frac{\ln (1+|u|)}{2 n\left(1+\exp \left(i+t^{2}\right)\right)}, \quad g_{1, i}(t, u, v)=\frac{\ln |u|}{\left(10 n \pi \frac{4^{\frac{2 i-1}{2}}}{\Gamma\left(\frac{2 i+1}{2}\right)}\right)\left(1+t^{2}\right)}, \quad i=1,2, \ldots, n, \\
k_{2, j}(t, u, v)=\frac{\ln (1+|v|)}{2 n(1+\ln |v|}, \quad g_{2, j}(t, u, v)=\frac{\left(2 n \pi^{2} \frac{4^{\frac{2 i+1}{4}}}{\Gamma\left(\frac{2 i+5}{4}\right)}\right)\left(1+t^{2}\right)}{\left.\left(\ln ^{2}\right)\right)}, \quad j=1,2, \ldots, n, \\
f_{1, i}(t, u, v)=4 \frac{\left(\tan ^{-1}(i+t+u)\right)^{2}+\left(\cot ^{-1}(i+t+v)\right)^{2}}{\pi}, \quad i=1,2, \ldots, n, \\
f_{2, j}(t, u, v)=\frac{\left(\sin ^{-1}(j+t+u)\right)^{2}+\left(\cos ^{-1}(j+t+v)\right)^{2}}{8}, \quad j=1,2, \ldots, n,
\end{array}\right.
$$

and after a direct calculation, we have

$$
\begin{aligned}
& \sup \left\{f_{1, i}(t, u, v) \mid t \in \mathbb{J}, u, v \in C(\mathbb{R}), i=1,2, \ldots, n\right\}=5 \pi, M_{i}=5 \pi \frac{4^{\frac{2 i-1}{2}}}{\Gamma\left(\frac{2 i+1}{2}\right)}, \quad i=1,2, \ldots, n, \\
& \sup \left\{f_{2, j}(t, u, v) \mid t \in \mathbb{J}, u, v \in C(\mathbb{R}), j=1,2, \ldots, n\right\}=\pi^{2}, M_{i}=\pi^{2} \frac{4^{\frac{2 i+1}{4}}}{\Gamma\left(\frac{2 i+5}{4}\right)}, \quad j=1,2, \ldots, n .
\end{aligned}
$$

By means of above setting all of conditions $\left(A_{1}\right)-\left(A_{4}\right)$ hold. So according on the Theorem 3.1 we deduce that coupled hybrid system of FQIEs (4.1) has at least one positive solution in $S$.

\section{Acknowledgement}

The authors express their sincere gratitude to anonymous referee for insightful reading the manuscript and constructive comments that led to the improvement the quality of paper.

\section{References}

[1] B. C. Dhage, On a fixed point theorem in Banach algebras with applications, Appl. Math. Lett., 18 (2005), 273-280.

[2] B. C. Dhage and S. B. Dhage, Approximating positive solutions of PBVPs of nonlinear first order ordinary quadratic differential equations, Appl. Math. Lett., 46 (2015), 133-142.

[3] B. C. Dhage, Quadratic perturbations of periodic boundary value problems of second order ordinary differential equations, Differ. Equ. Appl., 2 (2010), 465-486.

[4] B. C. Dhage, Hybrid fixed point theory in partially ordered normed linear spaces and applications to fractional integral equations, Differ. Equ. Appl., 5 (2013), 155-184.

[5] B. C. Dhage and V. Lakshmikantham, Basic results on hybrid differential equations, Nonlinear Anal. Hybrid Syst., 4 (2010), 414-424. 
[6] B. C. Dhage, J. Henderson and S. K. Ntouyas, Periodic boundary value problems of first order differential equations in Banach algebras, J. Nonlinear Funct. Anal. Differ. Equ., 1 (2007), 103-120.

[7] B. C. Dhage and S. B. Dhage, Approximating solutions of nonlinear PBVPs of hybrid differential equations via hybrid fixed point theory, Indian J. Math., 57(2015), 103-119.

[8] B. C. Dhage, S. B. Dhage and S. K. Ntouyas, Approximating solutions of nonlinear hybrid differential equations, Appl. Math. Lett., 34 (2014), 76-80.

[9] B. C. Dhage, Global attractivity results for comparable solutions of nonlinear hybrid fractional integral equations, Differ. Equ. Appl., 6 (2014), 165-186.

[10] B. C. Dhage, Partially condensing mappings in partially ordered normed linear spaces and applications to functional integral equations, Tamkang J. Math., 45 (2014), 397-426.

[11] B. C. Dhage, On some nonlinear alternatives of Leray-Schauder type and functional integral equations, Arch. Math., 42 (2006), 11-23.

[12] B. C. Dhage, Operator theoretic techniques in the theory of nonlinear hybrid differential equations, Nonlinear Anal. Forum, 20 (2015), 15-31.

[13] Ahmed El-Sayed, Hind Hashem, Existence results for nonlinear quadratic integral equations of fractional order in Banach algebra, Fract. Calc. Appl. Anal., 16 (2013), 816-826, DOI: 10.2478/s13540-013-0051-6.

[14] Kazem Ghanbari and Yousef Gholami, Existence and multiplicity of positive solutions for a coupled system of perturbed nonlinear fractional differential equations, Comput. Methods Differ. Equ., 1 (2013), 39-54.

[15] Kazem Ghanbari and Yousef Gholami, Existence and multiplicity of positive solutions for m-point nonlinear fractional differential equations on the half line, Electron. J. Differential Equations, 238 (2012), 1-15.

[16] Kazem Ghanbari and Yousef Gholami, Existence and nonexistence results of positive solutions for nonlinear fractional eigenvalue problem, J. Fract. Calc. Appl., 4 (2013), 1-12.

[17] Kazem Ghanbari, Yousef Gholami and Hanif Mirzaei, Existence and multiplicity results of positive solutions for boundary value problems of nonlinear fractional differential equations, Dyn. Contin. Discrete Impuls. Syst. Ser. A Math. Anal., 20 (2013), 543-558.

[18] Yousef Gholami and Kazem Ghanbari, On solvability of fractional coupled hybrid systems, Asia Pacific Journal of Mathematics, 2 (2015), 54-68.

[19] Yousef Gholami, Existence results of positive solutions for boundary value problems of fractional order with integro-differential boundary conditions, Differ. Equ. Appl., 6 (2014), 59-72.

[20] Yousef Gholami, Existence of an unbounded solution for multi-point boundary value problems of fractional differential equations on an infinite domain, Fractional. Differ. Calc., 4 (2014), 125-136.

[21] L. P. Lebedev, I. I. Vorovich and G. M. L. Gladwell, Functional Analysis (Applications in Mechanics and Inverse Problems, $2^{\text {nd }}$ Edition), Kluwer Academic Publishers, 2002.

[22] X. Long, X. Zheng and L. Li, Existence of periodic solutions for a class of functional integral equations, Electron. J. Qual. Theory Differ. Equ., 57 (2012), 1-11.

[23] A. A. Kilbas, H. M. Srivastava and J. J. Trujillo, Theory and Applications of fractional Differential Equations, North-Holland mathematics studies, Elsevier science, 204 (2006).

[24] H. K. Pathak and R. R. Lopez, Existence and approximation of solutions to nonlinear hybrid ordinary differential equations, Appl. Math. Lett., 39 (2015), 101-106.

[25] Ivo Petras, Fractional-order nonlinear systems, modeling, analysis and simulation, Nonlinear Physical Science, Springer, 2011.

[26] I. Podlubny, Fractional Differential Equations, Mathematics in Science and Applications, Academic Press, New York, 19 (1999).

[27] J. M. Wing and J. D. Zahrt, A primer on integral equations of first kind, The problem of deconvolution and unfolding, SIAM, Philadelphia, 1923.

Department of Mathematics, Sahand University of Technology, P. O. Box: 51335-1996, Tabriz, Iran.

E-mail: kghanbari@sut.ac.ir

Department of Mathematics, Sahand University of Technology, P. O. Box: 51335-1996, Tabriz, Iran.

E-mail: y_gholami@sut.ac.ir 